\title{
Critical Phenomena in Continuous Dimension
}

\author{
H. Ballhausen, J. Berges, and C. Wetterich \\ Institute for Theoretical Physics, Heidelberg University \\ Philosophenweg 16, 69120 Heidelberg, Germany
}

\begin{abstract}
We present a calculation of critical phenomena directly in continuous dimension $d$ employing an exact renormalization group equation for the effective average action. For an Ising-type scalar field theory we calculate the critical exponents $\nu(d)$ and $\eta(d)$ both from a lowest-order and a complete first-order derivative expansion of the effective average action. In particular, this can be used to study critical behavior as a function of dimensionality at fixed temperature.
\end{abstract}

PACS numbers: $05.10 . \mathrm{Cc}, 05.70 . J \mathrm{k}, 11.10 \mathrm{Kk}$

\section{INTRODUCTION}

One of the important quantitative successes of statistical physics is the precise understanding of critical phenomena based on the renormalization group [1]. Universal properties near second-order phase transitions are found to be insensitive to the details of the underlying theory and only to depend on symmetry, field content and the dimensionality of space $d$. The crucial dependence of critical behavior on $d$ can be readily observed from the fact that no phase transition at nonzero temperature or, equivalently, no corresponding renormalization group fixed point exists for $d=1$. For higher dimensions phase transitions can occur, depending on the model. For instance, simple Ising-type one-component models can exhibit an infinite number of multi-critical points in $d=2$ 2], a critical "Wilson-Fisher" or a tri-critical fixed point in $d=3$, and a trivial "Gaussian" fixed point for $d=4$.

Continuity in the dimensionality of space is a crucial ingredient for some of the standard methods for the description of critical phenomena. In particular the extrapolation from $d=4$ to lower dimension $\left(4-\epsilon^{-}\right.$ expansion [1]) is one of the most frequently applied methods for studying the non-perturbative dynamics near a non-trivial fixed point of the renormalization group equation. In this language the strong infrared divergences encountered by perturbation theory at fixed dimension are generated by an expansion around the "wrong" (Gaussian) fixed point. The $\epsilon$-expansion is used to interchange limits which allows one to follow "perturbatively" the relevant fixed point. The involved extrapolation in the dimension $d$ is a crucial ingredient for this procedure. In particular, the typical expansion parameter $\epsilon=1$ is not small and improvement procedures such as Borel transformation and conformal mapping are required to obtain accurate results [3]. A direct calculation in continuous dimensions would provide important insight concerning the validity of such an approach.

In this work we calculate critical phenomena for continuous dimension $d$ within a one-component scalar field theory with quartic self-interaction. Computations in arbitrary dimension can be performed [4, 5] using the effective average action $\Gamma_{k}, 6$, 7], which is infrared regulated by a momentum cutoff. The change of $\Gamma_{k}$ with the scale $k$ is described in terms of an exact flow equation [6]:

$$
\partial_{k} \Gamma_{k}[\phi]=\frac{1}{2} \operatorname{Tr}\left[\left(\Gamma_{k}^{(2)}[\phi]+R_{k}\right)^{-1} \partial_{k} R_{k}\right],
$$

where $\Gamma_{k}^{(2)}[\phi]$ is the exact inverse propagator and $R_{k}$ denotes the cutoff function. We investigate the theory in complete first-order in a derivative expansion, where

$$
\Gamma_{k}=\int \mathrm{d}^{d} x\left[U_{k}(\rho)+\frac{1}{2} Z_{k}(\rho) \partial_{\mu} \phi \partial^{\mu} \phi\right]
$$

with $\rho=\phi^{2} / 2$. Critical phenomena can be described from the flow of the most general effective potential $U_{k}(\rho)$ consistent with the symmetries, as well as the wave function renormalization $Z_{k}(\rho)$ []. This approach allows one to perform direct calculations for a given continuous dimension parameter $d$. In particular, this can be used to study critical behavior as a function of $d$ at fixed temperature.

\section{EVOLUTION EQUATIONS}

For the truncation (2) the exact renormalization group equation (II) is reduced to partial differential equations for $U_{k}(\rho)$ and $Z_{k}(\rho)$. These equations can be found in Refs. [7, 8]. For the cutoff function we employ $[9]$

$$
R_{k}=Z_{k}\left(k^{2}-p^{2}\right) \Theta\left(k^{2}-p^{2}\right)
$$

with $Z_{k}=Z_{k}\left(\rho=\rho_{0}\right)$ evaluated at vanishing external momentum. The flow of the function $Z_{k}$ is determined by the anomalous dimension $\eta=-\partial_{t} \ln Z_{k}$. Our quantitative results are based on a complete numerical solution of the equations for the first-order derivative expansion. For comparison, we solve in addition a simpler approximation with a field independent wave function renormalization $Z_{k}$. For our analytical discussion we concentrate 
on the latter, for which the flow of the rescaled effective potential $u(\tilde{\rho})=k^{-d} U_{k}$ as a function of the renormalized and dimensionless field variable $\tilde{\rho}=Z_{k} k^{2-d} \rho$ is described by

$$
\begin{aligned}
\partial_{t} u= & -d u+(d-2+\eta) \tilde{\rho} u^{\prime} \\
& +\frac{4 v_{d}}{d}\left(1-\frac{\eta}{d+2}\right) \frac{1}{1+u^{\prime}+2 \tilde{\rho} u^{\prime \prime}}, \\
\eta= & \frac{8 v_{d}}{d} \frac{\kappa\left(3 \lambda+2 \kappa u_{3}\right)^{2}}{(1+2 \kappa \lambda)^{4}},
\end{aligned}
$$

with $v_{d}^{-1}=2^{d+1} \pi^{d / 2} \Gamma(d / 2)$ and $u^{\prime}=\partial u / \partial \tilde{\rho}$. Here $t=\ln (k / \Lambda)$, where $\Lambda$ denotes a high momentum scale. Most of the relevant qualitative properties can be obtained from the $k$-dependence of the location $\kappa$ of the minimum of $u(\tilde{\rho})$. By taking the total $t$-derivative of $u^{\prime}(\tilde{\rho}=\kappa)=0$ the scale-dependence of the minimum for $\kappa>0$ is found to be:

$$
\begin{aligned}
\partial_{t} \kappa & =\beta_{\kappa}=-(d-2+\eta) \kappa+\frac{12 v_{d}}{d} N_{r}, \\
N_{r} & =\left(1-\frac{\eta}{d+2}\right) \frac{1+2 \kappa u_{3} /(3 \lambda)}{(1+2 \kappa \lambda)^{2}} .
\end{aligned}
$$

Here the couplings are related to $\tilde{\rho}$-derivatives of the rescaled potential evaluated at the minimum, i.e. $\lambda=$ $u^{\prime \prime}(\kappa)$ and $u_{3}=u^{\prime \prime \prime}(\kappa)$. Note that $\beta_{\kappa}$ is parametrized in terms of the couplings $\lambda$ and $u_{3}$. Implicitly, however, $\beta_{\kappa}$ depends on an infinite number of couplings since the correponding $\beta$-function for $\lambda$ depends on the higher couplings $u_{3}$ and $u_{4}=u^{(4)}(\kappa)$, the one for $u_{3}$ depends on $u_{4}$ and $u_{5}=u^{(5)}(\kappa)$ etc.

A second-order phase transition is characterized by a fixed point or scaling solution for which $\partial_{t} u^{\prime}=0$ and, in particular, $\beta_{\kappa}=0$. All renormalized dimensionless couplings become independent of the scale $k$ and take on their "fixed point values" $\kappa_{*}, \lambda_{*}, u_{3 *}$ etc. Typically, $\kappa$ corresponds to the relevant parameter such that critical behavior can be observed only for a fine-tuned initial value $\kappa(\Lambda) \simeq \kappa_{c}$ with $\left(\kappa(\Lambda)-\kappa_{c}\right) \sim\left(T_{c}-T\right)$. If $\kappa$ is the only relevant parameter the flow of all other couplings $\lambda, u_{3}$ and higher derivatives of the potential follow fixed "critical trajectories" $\lambda(\kappa, d), u_{3}(\kappa, d)$ - so-called partial fixed points. (More precisely, this holds if $T$ is in the vicinity of the critical temperature $T_{c}$ and $k$ is sufficiently below $\Lambda$.) Inserting the critical trajectories, $\beta_{\kappa}$ becomes only a function of $\kappa$ and $d$ :

$$
\beta_{\kappa}(\kappa, d) \equiv \beta_{\kappa}(\kappa, \lambda(\kappa, d), \ldots, d) .
$$

For arbitrary $d$ the critical behavior is then directly linked to the properties of $\beta_{\kappa}(\kappa, d)$ - in particular its zeros. The precise form of the critical trajectories $\lambda(\kappa, d)$ etc. is often not known analytically but it can be determined numerically. However, we will see that many crucial properties of $\beta_{\kappa}(\kappa, d)$ do actually not involve a very precise analytical understanding of the critical trajectories. We emphasize that the use of $\lambda(\kappa, d)$ etc. in the flow equations is valid only in the vicinity of the critical trajectory. The analytical discussion in the next section is therefore limited to the behavior close to criticality. The full numerical treatment below is not restricted to this.

\section{ASYMPTOTIC BEHAVIOR FOR LARGE $\kappa$}

As a general feature, $\beta_{\kappa}(\kappa, \lambda, \ldots, d)$ is analytic in all arguments except for $\lambda=0$. This does, however, not imply automatically that $\beta_{\kappa}(\kappa, d)$ is analytic since the solutions for the critical trajectories of $\lambda(\kappa, d)$ and the higher couplings can be non-analytic. For fixed $\kappa, \lambda$, etc. the function $\beta_{\kappa}$ decreases with increasing $d$. According to (6), (77) and (5) one has

$$
\beta_{\kappa}=\frac{12 v_{d}}{d} \text { for } \kappa=0 .
$$

To discuss $\beta_{\kappa}$ for $\kappa \rightarrow \infty$ we note that the leading behavior is given by

$$
\beta_{\kappa}=\left\{\begin{array}{cc}
(2-d) \kappa & \text { for } \kappa \rightarrow \infty, d>2 \\
c_{\infty}^{(d)} & \text { for } \kappa \rightarrow \infty, d \leq 2
\end{array} .\right.
$$

As is shown below, here $c_{\infty}^{(d)}$ is a constant which depends on $d$. In particular, in two dimensions one finds $c_{\infty}^{(2)}=-1 /(4 \pi)[8]$. For positive $c_{\infty}^{(d)}$ the asymptotic behavior can be related to a so-called "zero-temperature phase transition". In this case critical behavior can be associated with a fixed point for $\partial_{t} \kappa^{-1}=-\kappa^{-2} \beta_{\kappa}$ at $1 / \kappa=0$.

To establish the leading behavior (10) for $d<2$ we first note that the region of large $\kappa$ admits slowly evolving solutions if $\eta$ is near $2-d$. From Eqs. (6) and (5) one observes

$$
\kappa_{*}^{3} \lambda_{*}^{2}=\frac{2 v_{d}}{d(d+2)^{2}} \eta\left(\frac{d+2-\eta}{d-2+\eta}\right)^{2},
$$

which shows that the presence of fixed points in the region of large $\kappa^{3} \lambda^{2}$ requires values of $\eta$ close to $2-d$. We parametrize $\eta=2-d+\zeta / \kappa$, where $\zeta$ is a $\kappa$-independent constant. Introducing the notation $u(\tilde{\rho})=\tilde{\rho}^{-1 / 2} h(\tilde{\rho})$ one finds from Eq. (44):

$$
\begin{aligned}
\left.\partial_{t} h\right|_{\tilde{\rho}}= & -d h-\frac{\zeta}{2 \kappa} h+\frac{\zeta}{\kappa} \tilde{\rho} h^{\prime} \\
& +\frac{4 v_{d}}{d+2}\left(1-\frac{\zeta}{2 \kappa d}\right) \frac{1}{h^{\prime \prime}-\frac{h^{\prime}}{2 \tilde{\rho}}+\frac{h}{2 \tilde{\rho}^{2}}+\frac{1}{2 \sqrt{\tilde{\rho}}}} .
\end{aligned}
$$

Since we are interested in values for $\tilde{\rho}$ near $\kappa$ we consider the variable $x=\tilde{\rho}-\kappa$ and neglect terms suppressed by powers of $x / \kappa$ as well as $\zeta / \kappa$, i.e.

$$
\begin{aligned}
\left.\partial_{t} h\right|_{x} & =\left.\partial_{t} h\right|_{\tilde{\rho}}+h^{\prime} \partial_{t} \kappa \\
& =-d h+\frac{12 v_{d}}{d} N_{r} h^{\prime}+\frac{4 v_{d}}{(d+2) h^{\prime \prime}}-\zeta h^{\prime} .
\end{aligned}
$$


We observe that $\kappa$ does not appear anymore in (13) and consequently quantities like $h_{2} \equiv h^{\prime \prime}(x=0), h_{3} \equiv$ $h^{\prime \prime \prime}(x=0)$ are not expected to depend on $\kappa$. In leading order for $\kappa \rightarrow \infty$ we therefore have $\lambda \sim \kappa^{-1 / 2}, u_{3} \sim$ $\kappa^{-1 / 2}$ and $\lambda \kappa \sim \kappa^{1 / 2} \gg 1$ as well as $\kappa u_{3} \sim \kappa^{1 / 2} \gg \lambda$. In particular, $N_{r}$ is also independent of $\kappa$ and given by

$$
N_{r}=\frac{d}{3(d+2)} \frac{h_{3}}{h_{2}^{3}} .
$$

This shows that the leading behavior of $\beta_{\kappa}$ for $\kappa \rightarrow \infty$ and $d<2$ is described by

$$
\beta_{\kappa}=-\zeta+\frac{12 v_{d}}{d} N_{r}=c_{\infty}^{(d)} .
$$

Further information can be obtained from the scaling solution $\left.\partial_{t} h\right|_{x}=0$. (Note that the scaling solution for $h$ does not correspond to an exact fixed point since $\kappa$ evolves according to (15).) At the potential minimum we have $h^{\prime}(x=0)=0$ up to corrections $\sim 1 / \kappa$. From (13) one then finds

$$
h_{2}=\frac{4 v_{d}}{d(d+2) h_{0}} .
$$

The integration constant $h_{0}=h(x=0)$ has to be chosen such that the lowest order relation for the anomalous dimension, $\eta_{0}=2-d$, holds. According to Eq. (5) at this order

$$
\eta_{0}=\frac{18 v_{d}}{d^{3}}(d+2)^{2} h_{2}^{2} N_{r}^{2} \stackrel{!}{=} 2-d
$$

which fixes $N_{r}$ as a function of $h_{2}$ :

$$
N_{r}= \pm \sqrt{\frac{(2-d) d}{2 v_{d}}} \frac{d}{3(d+2) h_{2}}
$$

and $h_{3}$ by (14). Finally, $h_{2}$ is constrained by the requirement that the scaling solution should extend to the whole range $-\kappa<x<\kappa$. For $d \rightarrow 2$ one concludes from (18) that $N_{r} \rightarrow 0$. As a consequence, $h$ is determined by the motion of a particle in a logarithmic potential, i.e. for $d \rightarrow 2$ the lowest order scaling solution for (13) reads

$$
\left(h^{\prime}\right)^{2}=\frac{8 v_{d}}{d(d+2)} \ln \left(\frac{h}{h_{0}}\right) .
$$

It is striking that the simple equations (4), (5) and (6) reproduce correctly the low-temperature behavior of the exact Ising model for $d=1$, which is characterized by $\eta=1$ and essential scaling: For $T \rightarrow 0$ from the leading behavior (15) one has

$$
\frac{\partial \kappa}{\partial k}=\frac{c_{\infty}^{(1)}}{k} .
$$

Associating the scale $k_{s}$ where $\kappa\left(k_{s}\right)=0$ to the inverse correlation length $\xi^{-1}$ up to a factor one finds

$$
\xi \Lambda \sim \exp \left(\frac{\kappa(\Lambda)}{c_{\infty}^{(1)}}\right) \sim \exp (2 J / T)
$$

with $\kappa(\Lambda)=b / T, b=2 c_{\infty}^{(1)} J$.

\section{NON-ZERO TEMPERATURE PHASE TRANSITION}

From the asymptotic behavior discussed above one can draw a number important conclusions about the presence of a phase transition at non-zero temperature, characterized by a finite value $\kappa_{*}$. We note that a positive value of $\beta_{\kappa}$ for $\kappa=0$ and negative values for large $\kappa$ require the $\beta$-function to vanish at an intermediate $\kappa=\kappa_{*}$. Since $c_{\infty}^{(2)}=-1 /(4 \pi)$ Eq. (10) immediately tells us that for all $d \geq 2$ a finite value $\kappa_{*}$ exists for which $\beta_{\kappa}=0$. For instance, in $d=3$ the corresponding phase transition is described by the well-known Wilson-Fisher fixed point in the presence of only one relevant parameter [3].

From the exact solution of the one-dimensional Isingmodel one has $c_{\infty}^{(1)}>0$ and $\beta_{\kappa}$ is above a positive constant for all $\kappa$. Therefore, no fixed point with a finite $\kappa_{*}$ occurs in $d=1$. If the $\beta$-function depends continuously on $d$ we conclude that the zero-temperature transition and the absence of a fixed point with finite $\kappa_{*}$ extend to a finite range of $d>1$. (It is crucial in this respect that the limiting value $c_{\infty}^{(1)}$ is strictly positive.) As a consequence, the lower critical dimension, below which phase transitions at nonzero temperature are absent, has to be larger than one.

The lower bound on the critical dimension, $d_{\text {low }}>1$, can be supplemented by an upper bound using similar continuity arguments. For this we note that the correlation length exponent $\nu$ at an infrared-unstable fixed point is given by the first derivative of $\beta_{\kappa}(\kappa, d)$ at $\kappa_{*} \neq 0$ :

$$
\nu^{-1}=-\left.\frac{\partial \beta_{\kappa}(\kappa, d)}{\partial \kappa}\right|_{\kappa_{*}} .
$$

For finite $\nu$ this yields the usual power law for the correlation length, $\xi \sim\left|T-T_{c}\right|^{-\nu}$ [3]. For a vanishing first derivative the exponent $\nu$ diverges. In this case the power law is replaced by essential scaling. A finite value for $\nu$ corresponds to nonzero negative $\partial \beta_{\kappa} / \partial \kappa_{\mid \kappa_{*}}<0$. Since the derivative of the $\beta$-function (and therefore $\nu$ ) depends continuously on $d$, we conclude that for finite $\nu$ at a given $d$ also a finite neighborhood in $d$ exhibits a phase transition. At the lower critical dimension, below which no phase transition with a finite $\kappa_{*}$ occurs, either $\kappa_{*} \rightarrow \infty$ or $\nu$ must diverge and $d_{\text {low }}$ is characterized by essential scaling. The finiteness of $\nu$ for $d=2$ follows from universality and the exact value for the Ising model, $\nu=1[3]$. Therefore $\nu$ must also remain finite in a finite range $d<2$. This continuity argument therefore implies $d_{\text {low }}<2$.

We emphasize that for a second-order phase transition, apart from a vanishing $\beta_{\kappa}$-function, all couplings have to take on fixed-point values and the critical trajectories $\lambda(\kappa, d)$ etc. have to be well defined. In particular, the character of a fixed point is influenced by the stability properties with respect to the other couplings. 


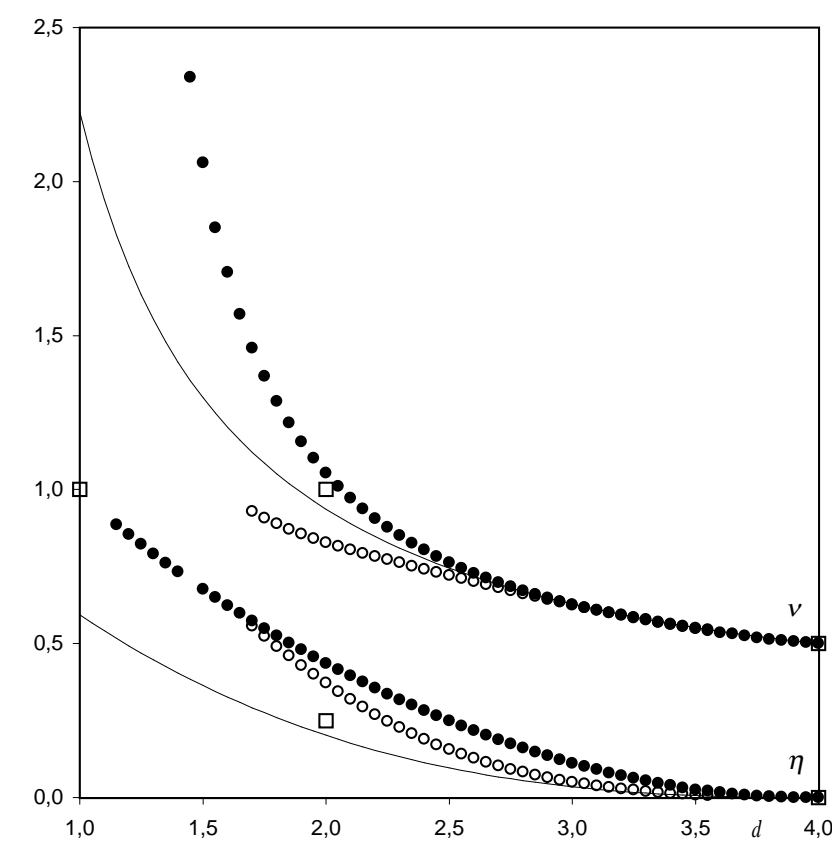

FIG. 1: The critical correlation length exponent $\nu$ and anomalous dimension $\eta$ as a function of the dimension $d$ for two different approximations: complete first-order derivative expansion (open circles) and for a field-independent wave function renormalization (full circles). We also show the results of the $\epsilon$-expansion up to $\epsilon^{5}$ (solid line) [8], which are plotted for the range $0 \leq \epsilon \leq 3$ for illustration, along with available exact results (open squares).

To establish this we turn to the numerical solution of the full first-order derivative expansion, as well as for the approximation with a field-independent wave function renormalization as discussed above. Since the approximations include a most general potential term $u(\tilde{\rho})$ the description takes into account an "infinite number of couplings" $u_{n}=\left.u^{(n)}(\tilde{\rho})\right|_{\tilde{\rho}=\kappa}$.

We numerically solve the evolution equations, which are given e.g. in Eqs. (3.8) and (3.10) of Ref. [11], for the one-component theory to first order in the derivative expansion. The equations were discretized on a grid for the variable $\tilde{\rho}$ with varying grid-size (typically thirty points and much higher resolution in lower dimensions). The resulting ordinary differential equations were solved using a fifth-order Runge-Kutta algorithm and a fourth-order Cash-Karp stepping routine. The relative local errors of the integration routine were below $10^{-6}$. Results for sample exponents in various dimensions were cross-checked with different numerics (in particular, the integration of the flow equation of the potential $u(\tilde{\rho})$ instead of its first derivative $u^{\prime}(\tilde{\rho})$, as well as a fixed grid instead of running discretization points $\sim \kappa)$. The quantitative differences in the exponents using the different numerics were manifest only in the third to fifth significant digit.

The results for the critical exponent $\nu(d)$ and the anomalous dimension $\eta(d)$ are plotted in Fig. 1 for the two different employed approximations. For comparison we also include the results obtained from the expansion around $4-\epsilon$ dimensions up to $\epsilon^{5}[3,10]$ as well as available exact results. The results from the $\epsilon$-expansion have been plotted for values as large as $\epsilon=3$ for illustrational purposes though it is typically employed only for $\epsilon=1$. In contrast, we emphasize that our results correspond to direct calculations for the corresponding dimensions. In particular, they do not involve any improvement procedure such as Borel transformation and conformal mapping employed for the expansion around $4-\epsilon[3,10]$.

To numerically obtain $\nu(d)$ we calculate a series of near-critical trajectories with varying $\left(\kappa(\Lambda)-\kappa_{c}\right) \sim$ $\left(T_{c}-T\right)$. These calculations become time intensive for low dimensions, and grow substantially for the more sophisticated approximation with a field-dependent $Z_{k}(\tilde{\rho})$. This is why we have computed $\nu(d)$ only down to about $d=1.5$ in Fig. 1 In contrast, the anomalous dimension $\eta$ can be directly infered from the critical trajectory and is presented in Fig. 1 for even lower dimensions.

As $d$ is lowered from the upper critical dimension $d=4$ both $\nu(d)$ and $\eta(d)$ grow. One observes a very good agreement of the results from our first-order derivative expansion and the $\epsilon$-expansion results down to about $d=3$. At $d=2$ we can compare with the exact values $\nu=1$ and $\eta=0.25$. The renormalization group results for $\nu$ with the $\Theta$-cutoff function (31) show a rather strong dependence on the approximation around $d=2$. The strong increase of $\nu(d)$ as $d$ is further lowered sets in much earlier for the approximation with a field-independent wavefunction renormalization. Interestingly, for the anomalous dimension the dependence on the approximation is diminished for lower dimensions. In particular, we observe that already the less sophisticated approximation approaches the exact one-dimensional result. This may be related to fact that in one dimension the propagator shows a simple quadratic dependence on momenta for $\eta=1$ [15].

\section{CRITICAL BEHAVIOR AS A FUNCTION OF DIMENSIONALITY}

We point out that critical behavior can also be studied as a function of $d$ for fixed $\kappa(\Lambda)$ or, equivalently, temperature $T$. In the critical region we find that for fixed $T$ the correlation length varies with $d$ as

$$
\xi \sim\left|d_{c}-d\right|^{-\nu} .
$$

Here $d_{c}$ is the dimension for which $T$ becomes the critical temperature and $\nu=\nu\left(d_{c}\right)$ is the same critical exponent as computed above for the variation with $T$ for fixed $d$. This property can be understood as a simple consequence of continuity. Assume some fixed point where $\beta_{\kappa}\left(\kappa_{*}\left(d_{c}\right), d_{c}\right)=0$ and $\left(\partial \beta_{\kappa} / \partial \kappa\right)\left(\kappa_{*}\left(d_{c}\right), d_{c}\right)=-\nu^{-1}\left(d_{c}\right)$ 
with finite critical exponent $\nu\left(d_{c}\right)$. Starting with critical initial values $\left(\kappa_{*}\left(d_{c}\right), d_{c}\right)$ at some scale $\bar{\Lambda}$ the correlation length $\xi$ diverges. For initial values $\left(\kappa_{*}\left(d_{c}\right)-\Delta, d_{c}\right)$ we have the well-known critical behavior $(\Delta>0$ in the symmetric phase) $\xi\left(\Delta, d_{c}\right) \sim \Delta^{-\nu\left(d_{c}\right)}$. Continuity tells us that for $d=d_{c}-\Delta_{d}$ there is a new fixed point with $\beta_{\kappa}\left(\kappa_{*}\left(d_{c}-\Delta\right), d_{c}-\Delta\right)=0$. Instead of varying $\Delta$ at fixed $d_{c}$ one may keep a fixed $\kappa(\Lambda)=\kappa_{*}\left(d_{c}\right)$ and vary $\Delta_{d}$. With $\kappa_{*}\left(d_{c}\right)=\kappa_{*}\left(d_{c}-\Delta_{d}\right)-\tilde{\Delta}$ this yields $\xi\left(0, d_{c}-\Delta_{d}\right) \sim \tilde{\Delta}^{-\nu\left(d_{c}-\Delta_{d}\right)}$. (Note that $\Delta_{d}>0$ for the symmetric phase.) For $\Delta_{d} \rightarrow 0$ we can relate $\tilde{\Delta}$ to $\Delta_{d}$

$$
\tilde{\Delta}=-\frac{\partial \kappa_{*}}{\partial d}\left(d_{c}\right) \Delta_{d}=-\nu\left(d_{c}\right) \frac{\partial \beta_{\kappa}}{\partial d}\left(\kappa_{*}\left(d_{c}\right), d_{c}\right) \Delta_{d}
$$

Generically one has $\partial \beta_{\kappa} / \partial d>0$ and we conclude that the dependence of $\xi$ on $d$ at the critical point $\kappa_{*}\left(d_{c}\right)$ is characterized by the standard critical exponent $\nu=\nu\left(d_{c}\right)$, which agrees with the results from the numerical solution to very good accuracy. This is investigated for the corresponding $N$-component model in Ref. [12]. We recall, however, that this argument is only valid for finite $\kappa_{*}$ and away from essential scaling at $d_{\text {low }}$.

\section{CONCLUSIONS}

We have presented a direct calculation of critical phenomena in continuous dimension emloying a complete first-order derivative expansion. The results for the critical exponents show good agreement with those found from the $\epsilon$-expansion for not too small dimensions down to about $d=3$. The deviations grow substantially for lower dimensions. To get an idea about the reliability of our results we have re-calculated the exponents using a less sophisticated approximation with a field-independent wave-function renormalization. It is striking to observe that though the critical behavior depends comparably strongly on the employed approximation scheme for intermediate values of $d$, this has only a small effect on some nontrivial properties at sufficiently low dimensions: even the less sophisticated approximation is sufficient to approach the exact result for the anomalous dimension $\eta=1$ in $d=1$. It has recently been demonstrated for the three-dimensional one-component model that the next order $\left(\partial^{4}\right)$ of the derivative expansion leads to significantly improved results 13. compared with lower orders calculations. It would be very interesting to investigate the apparent convergence of the derivative expansion for lower dimensions as well.
We have pointed out that with the employed methods one can use the dimensionality $d$ as a "relevant" parameter to study deviations from criticality. For fixed temperature $T$ the deviations from the critical dimension $d_{c}$ are described by the same universal critical exponents which parametrize the deviations from $T_{c}$ for fixed $d$. So far we are not aware of any clear experimental application of the calculated critical phenomena in fractional dimensions. However, an effectively similar behavior may be observed e.g. for the magnetic properties of thin films, showing a dimensional crossover from three- to two-dimensional scaling properties by varying the film thickness 14].

We thank Matthieu Tissier for helpful discussions.

[1] K. G. Wilson and J. B. Kogut, Phys. Rept. 12 (1974) 75.

[2] See e.g. T. R. Morris, Phys. Lett. B 345 (1995) 139 arXiv:hep-th/9410141.

[3] See e.g. J. Zinn-Justin, Quantum Field Theory and Critical Phenomena, Clarendon Press, Oxford; ibid. Phys. Rept. 344 (2001) 159 arXiv:hep-th/0002136.

[4] K. I. Aoki, K. i. Morikawa, W. Souma, J. i. Sumi and H. Terao, Prog. Theor. Phys. 95 (1996) 409 arXiv:hep-ph/9612458.

[5] M. Tissier, D. Mouhanna and B. Delamotte, Phys. Rev. B 61 (2000) 15327 arXiv:cond-mat/9908352.

[6] C. Wetterich, Phys. Lett. B 301 (1993) 90.

[7] J. Berges, N. Tetradis and C. Wetterich, Phys. Rept. 363 (2002) 223 arXiv:hep-ph/0005122.

[8] G. von Gersdorff and C. Wetterich, Phys. Rev. B 64 (2001) 054513 arXiv:hep-th/0008114.

[9] D. F. Litim, Phys. Rev. D 64 (2001) 105007 arXiv:hep-th/0103195.

[10] H. Kleinert, J. Neu, V. Schulte-Frohlinde, K. G. Chetyrkin and S. A. Larin, Phys. Lett. B 272 (1991) 39 [Erratum-ibid. B $\mathbf{3 1 9}$ (1993) 545] arXiv:hep-th/9503230.

[11] C. Wetterich, Int. J. Mod. Phys. A 16 (2001) 1951 arXiv:hep-ph/0101178.

[12] H. Ballhausen, in preparation.

[13] L. Canet, B. Delamotte, D. Mouhanna and J. Vidal, Phys. Rev. B 68 (2003) 064421 arXiv:hep-th/0302227.

[14] Y. Li and K. Baberschke, Phys. Rev. Lett. 68 (1992) 1208.

[15] The exact propagator $G$ of the one-dimensional Ising model in the zero temperature limit is given in Fourier space by $G(q)=f(q \xi) / q^{2-\eta}=f(q \xi) / q \sim \xi^{-1} /\left(q^{2}+\xi^{-2}\right)$ with $f(q \xi) \sim(q \xi)^{-1} /\left(1+(q \xi)^{-2}\right)$. 